ISSN 1808-3765

\title{
ANÁLISE MULTIVARIADA NA DETERMINAÇÃO DO RISCO DE EROSÃO EM SOLOS SOB IRRIGAÇÃO.
}

\author{
Célia Regina Paes Bueno ${ }^{1}$; Christiano Luna Arraes ${ }^{1}$; Gener Tadeu Pereira ${ }^{2}$; José \\ Eduardo Cora ${ }^{1}$; Sergio Campos ${ }^{3}$ \\ ${ }^{I}$ Departamento de Solos e Adubos, Faculdade de Ciências Agrárias e Veterinária, Universidade Estadual \\ Paulista, Jaboticabal,SP.crbueno@fcav.unesp.br \\ ${ }^{2}$ Departamento de Ciências Exatas, Faculdade de Ciências Agrárias e Veterinária, Universidade Estadual \\ Paulista, Jaboticabal, SP \\ ${ }^{3}$ Departamento de Engenharia Rural, Faculdade de Ciências Agronômicas, Universidade Estadual Paulista, \\ Botucatu, SP
}

\section{RESUMO}

O objetivo deste trabalho foi abordar a utilização de técnicas de análise multivariada na discriminação do risco de erosão dos solos, sob pivô central, em diferentes classes de solos, relevo, uso e manejo. A área de estudo de 33 ha localizada na região de Carmo de Rio Claro, MG, sob pivô central, vem sendo cultivada com feijão, milho e café por um período de 7 anos. As amostragens foram feitas a intervalos regulares de $10 \mathrm{~m}$ na profundidade de 0,00 $0,20 \mathrm{~m}$ em uma transeção de $1050 \mathrm{~m}$, perfazendo 59 amostras. Os parâmetros risco de erosão (A), potencial natural de erosão (PN) e expectativa de erosão (EE) foram avaliados por análise multivariada. A aplicação da análise multivariada mostrou uma boa associação entre os agrupamentos formados e os diferentes tipos de solos e, juntamente com os componentes principais, permitiram identificar dois grupos de maiores e menores perdas de solo, evidenciando que as áreas de maiores expectativas de perdas de solo estão correlacionadas com a classe de solo, o relevo e manejo do solo. O potencial natural da erosão do solo foi um fator importante para determinar os diferentes grupos. A análise multivariada mostrou que 98 $\%$ das variáveis foram classificadas dentro dos grupos e que estes pelo potencial erosivo requerem programas de manejo e conservação do solo.

UNITERMOS: Análise multivariada, componentes principais, solos, Equação Universal de Perdas de Solo.

\section{BUENO, C. R. P.; ARRAES, C. L.; PEREIRA. G.T.; CORÁ. J.E.; CAMPOS, S. MULTIVARIANCE ANALYSIS ON EROSION RISK DETERMINATION IN SOIL UNDER IRRIGATION.}

\section{ABSTRACT}

The objective of this work was to verify the application of cluster analysis to evaluate soil erosion risk for different soil classes, soil slopes and soil managements. The study was conducted in a 33 ha section of a large field located in Carmo do Rio Claro County, MG, Brazil. The field had been managed in a corn/bean rotation under conventional tillage and under coffee plantation for seven years, both under sprinkle irrigation. Soil samples were obtained at every $10 \mathrm{~m}$ at $0.20 \mathrm{~m}$ depth along a transect of $1050 \mathrm{~m}$. Soil erosion risk (A), 
natural potential erosion (PN), and erosion expectation (EE) were determined and submitted to a cluster and principal component analysis. The application of clustering analysis showed high correlation between the clusters and soil types. With clustering analysis plus principal components analysis, it was possible to identify groups of high and low soil erosion expectation, showing that the areas with higher soil erosion expectation are correlated to the soil class, soil slope and soil management. Among the studied variables, the natural potential erosion (PN) showed to be the most important factor to identify different soil erosion groups. The cluster analysis showed that $98 \%$ of the variables were classified within each group, and that they should be managed differently due to the soil erosive potential of each group,.

KEYWORDS: Cluster analysis, principal components analysis, soils, Universal Soil Loss Equation (USLE)

\section{INTRODUÇÃO}

A utilização de uma propriedade agrícola é, em geral, não planejada, contrariando na maioria das vezes, a sua real capacidade de uso, podendo levar a uma manifestação do processo erosivo do solo em intensidade e velocidade que ultrapassam os limites de tolerância. O processo erosivo consiste na perda de partículas que se desagregam do solo e são carregadas, levando junto os nutrientes, portanto a fertilidade do solo.

As taxas de erosão em agrossistemas tropicais são geralmente maiores que a formação do solo. Se a erosão superar a formação do solo o sistema agrícola não pode ser considerado sustentável, uma vez que os recursos naturais, como a fertilidade da superfície, são exauridos num futuro próximo (Sparovek \& Schnug, 2001). Esta taxa de erosão é determinada de uma maneira particular, na qual, os níveis das numerosas variáveis físicas e de manejo estão combinados nessa área (Wischmeier \& Smith, 1978).

Devido à dificuldade e os altos custos envolvidos em determinações diretas de perdas de solo, tem aumentado o uso de métodos indiretos de predições de perdas. A Equação Universal de Perdas de Solo (EUPS) é uma das ferramentas que vem sendo utilizada para estimar perdas de solo com considerável sucesso. A equação possibilita estabelecer paralelos com a suscetibilidade natural dos solos à erosão, quando a análise envolve apenas os fatores inerentes ao meio natural, e paralelos com riscos atuais de erosão, quando introduzidos os fatores antrópicos. Estes modificam as tendências naturais do meio a erosão, ou seja, alteram as perdas naturais do solo. As diferenças discriminam os riscos (IPT, 1988; Stein et al., 1987; Bueno \& Stein, 2004 ).

Tomazoni et al. (2005) estudando a sistematização dos fatores da EUPS em SIG para quantificação da erosão laminar na bacia do Rio Anta Gorda (PR), verificou que a erodibilidade varia para cada tipo de solo, pois mesmo que os fatores declividade, precipitação, cobertura vegetal e práticas conservacionistas fossem iguais em solos argilosos e arenosos, os últimos, devido às suas características físicas e químicas, são mais susceptíveis à erosão.

Para obter um grande número de informações sobre a conservação dos recursos naturais, os métodos de avaliação de perdas de solo são seguramente a melhor forma, pois possibilitam aos usuários empregarem técnicas e estabelecerem planejamentos para que possam prolongar, e até mesmo aumentar, a capacidade produtiva de suas terras. Estudar e colocar em práticas tecnologias disponíveis, dentre as quais a análise do comportamento dos 
parâmetros do risco de erosão seria uma forma de contribuir com a avaliação das perdas de solo.

A análise multivariada de componentes principais é utilizada para agrupar indivíduos com características similares e estudar suas correlações. Webster \& Oliver (1990) consideram a análise discriminante uma poderosa ferramenta para levantamentos de solos. O emprego desta análise pode ser verificado nos trabalhos de Demattê \& Garcia (1999) e Demattê \& Nanni (2003) que demostraram por meio desta análise a possibilidade de predizer classes de solos e/ou seus limites na paisagem; Kravchenko et al (2002) que a utilizou em levantamentos pedológicos e dos atributos do solo; Lima et al (2007) que utilizou quatro diferentes conjuntos de variáveis químicas e físicas para determinar a sustentatibilidade dos sistemas de cultivo orgânico e Valladares et al. (2008), que correlacionaram as semelhanças dos perfis de Organossolos, com base em atributos morfológicos, físicos e químicos. Com esses estudos pode-se explorar a existência ou não da dependência entre as variáveis analisadas para melhores interpretações e conclusões (Fikdalski et al, 2007).

Portanto, o objetivo deste trabalho foi abordar a utilização de técnicas de análise de agrupamentos na discriminação do risco de erosão dos solos, sob pivô central, em diferentes classes de solos, relevo, uso e manejo.

\section{MATERIAIS E MÉTODOS}

O estudo foi realizado em uma área localizada no município de Carmo do Rio Claro (MG), com longitude $46^{\circ} 07^{\prime} \mathrm{W}$ e latitude $20^{\circ} 58^{\prime} \mathrm{S}$, altitude média de $811 \mathrm{~m}$ e área de 33 ha, em relevo suave ondulado, sob pivô central e cultivada com milho, café e feijão, no sistema convencional de manejo de solo (Figura 1).

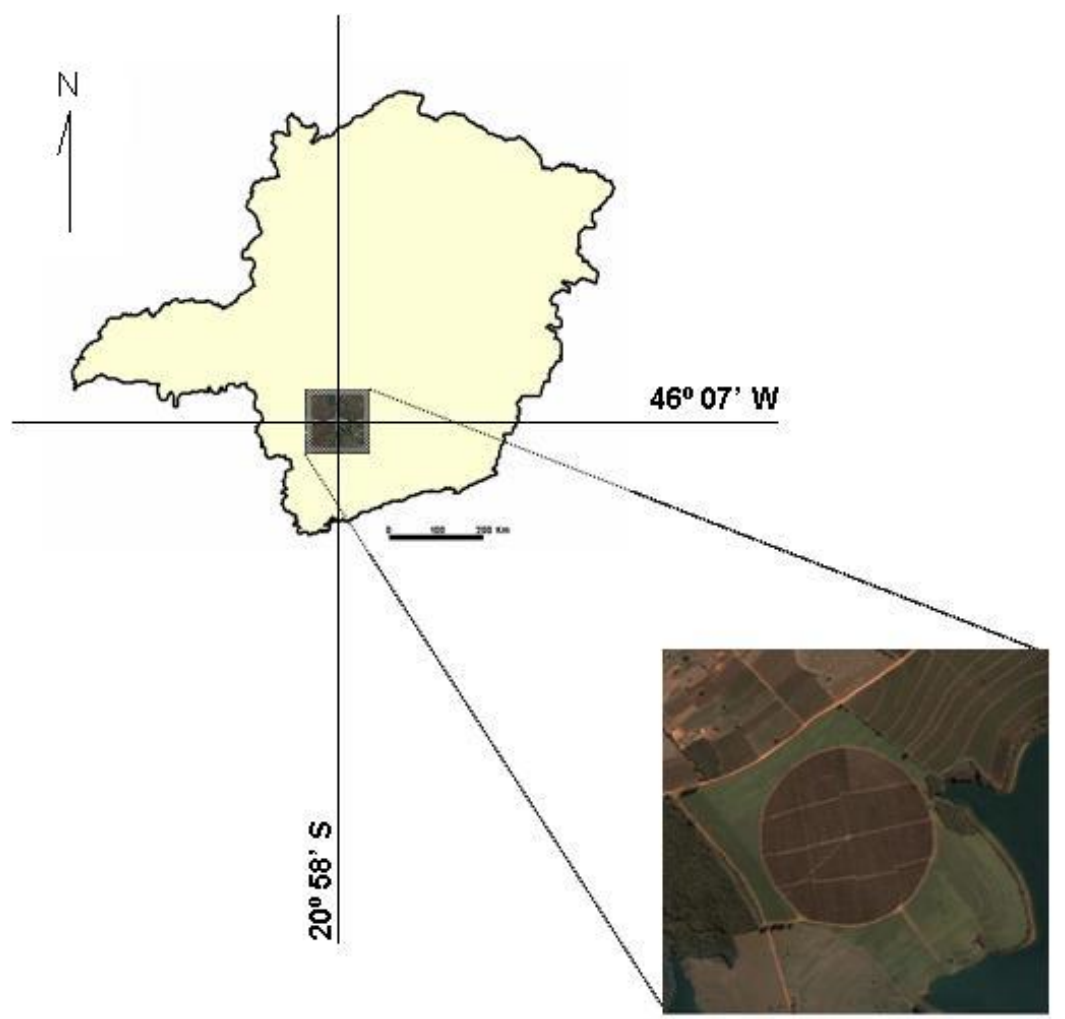

Figura 1. Localização da área de trabalho no município de Carmo do Rio Claro (MG). 
Os principais solos mapeados são Latossolo Vermelho eutrófico textura argilosa e Nitossolo Vermelho eutrófico textura franco argilo-arenosa. Foram coletadas amostras de solos, georreferenciadas, em uma transeção de $1050 \mathrm{~m}$ de extensão. A amostragem do solo foi realizada em intervalos regulares de $10 \mathrm{~m}$ na profundidade de $0,00-0,20 \mathrm{~m}$ em duas transeções, perfazendo um total de 59 pontos de amostragem (Figura 2), sendo 28 amostras de solo sob cultivo de milho e 31 amostras sob cultivo de feijão.

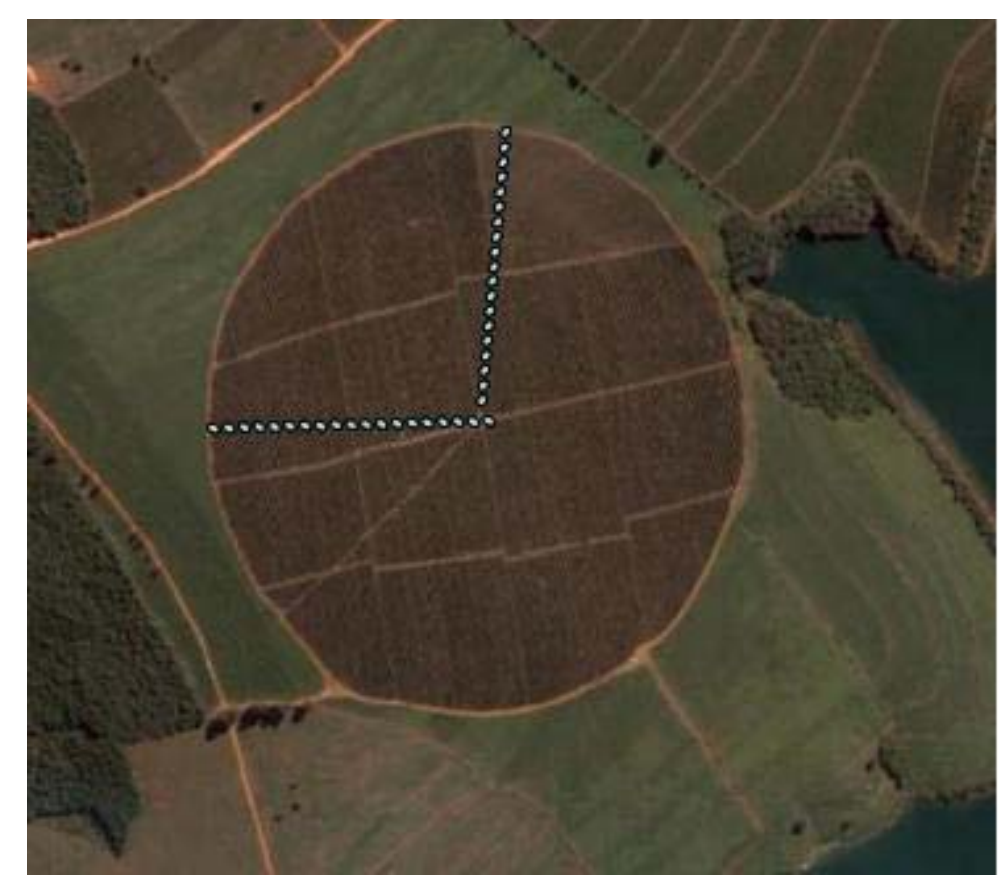

Figura 2. Vista do pivô central com transeções de coleta de amostras de solo.

Nas amostras foram determinadas a textura do solo, pelo método da pipeta (EMBRAPA, 1997) e o teor de matéria orgânica (MO) seguindo o método descrito por Raij et al. (1987).

A perda de solo (A), expressa em $\mathrm{t} \mathrm{ha}^{-1} \mathrm{ano}^{-1}$, foi estimada utilizando-se a Equação Universal de Perdas de Solo (EUPS), segundo Wischmeier \& Smith (1978), descrita na equação 1:

$$
\mathrm{A}=\mathrm{R} \text { K L S C P }
$$

O fator erosividade da chuva (R) expresso em MJ mm ha ${ }^{-1} \mathrm{~h}^{-1}$ obtido pelo meio da equação 2 proposta por Bertoni \& Lombardi Neto (1999):

$$
\mathrm{EI}_{\text {mensal }}=89,823\left(\mathrm{r}^{2} / \mathrm{P}\right)^{0,759}
$$

Sendo:

$\mathrm{EI}=$ média mensal do índice de erosão (MJ.mm/h.ha);

$\mathrm{r}=$ precipitação média mensal $(\mathrm{mm})$;

$\mathrm{P}=$ precipitação média anual $(\mathrm{mm})$.

Os dados médios de precipitação foram obtidos na estação meteorológica de Guaxupé e são provenientes da média de 62 anos. 
$\mathrm{O}$ fator da erodibilidade do solo $(\mathrm{K})$ expresso em $\mathrm{t}$ ha $\mathrm{h} \mathrm{ha} \mathrm{ha}^{-1} \mathrm{MJ}^{-1} \mathrm{~mm}^{-1}$, obtido pelo modelo proposto por Denardin (1990), equação 3:

$$
\mathrm{K}=7,48 \times 10^{-6} \mathrm{M}+4,48059 \times 10^{-3} \mathrm{P}-6,31175 \times 10^{-2} \mathrm{DMP}+1,039567 \times 10^{-2} \mathrm{R}
$$

Sendo:

$\mathrm{M}(\%)=$ areia fina + silte $\mathrm{x}(($ areia fina + silte $)+$ areia grossa $) ;$

$\mathrm{P}=$ valor da permeabilidade; $1=$ muito rápida, $2=$ rápida, $3=$ moderada, $4=$ lenta, $5=$ muito lenta, 6 = imperfeitamente drenado;

$\mathrm{DPM}=$ diâmetro médio ponderado das partículas de solo $(\mathrm{mm}) ; \mathrm{R}=$ areia grossa $\mathrm{x}$ teor de matéria orgânica/100.

O fator topográfico (LS) foi determinado pela equação 4, estimada por Bertoni \& Lombardi Neto (1999):

$$
\mathrm{LS}=0,00984 * \mathrm{C}^{0,63} * \mathrm{D}^{1,18}
$$

Sendo:

$\mathrm{C}=$ fator comprimento de rampa $(\mathrm{m})$; os valores de comprimento de rampa, segundo as classes de declividade, foram obtidos de forma similar aos obtidos por Sales (1998), que considera o comprimento de rampa correlacionado com as classes de solo e as classes de declividade.

$\mathrm{D}=$ grau do declive (\%); obtido com um módulo do programa Spring (2002), utilizando-se como entrada, os dados planialtimétricos gerados por interpolação no programa SURFER v.6.01 (Golden Software, 1995). Posteriormente, as classes de declividade obtidas foram reclassificadas adotando-se os seguintes intervalos: A de 0 a 3\%; B de 3 a 6\%; C de 6 a 9\%; D de 9 a $12 \%$ e $E>12 \%$.

O fator uso e manejo do solo (C) foi obtido pelas razões de perdas de solo de cada período, combinadas com dados relativos à chuva, estabelecido no campo no momento da amostragem onde foi observada a cultura atual para cada ponto amostrado (Bertoni \& Lombardi Neto, 1999).

O fator práticas conservacionistas $(\mathrm{P})$ foi obtido por meio da equação 5 , definida por Lagrotti (2000):

$$
P=0,69947-0,08991 \mathrm{D}+0,01184 \mathrm{D}^{2}-0,000335 \mathrm{D}^{3}
$$

Sendo:

$\mathrm{D}(\%)=$ Declividade.

O potencial natural de erosão (PNE) foi calculado pela equação 6:

Sendo:

$$
\mathrm{PNE}=\mathrm{R} \text { K LS }
$$

$\mathrm{PNE}=$ potencial natural de erosão $\left(\mathrm{t} \mathrm{ha}^{-1} \mathrm{ano}^{-1}\right)$;

$\mathrm{R}=$ erosividade da chuva $\left(\mathrm{MJ} \mathrm{mm} \mathrm{ha}^{-1}\right)$;

$\mathrm{K}=$ erodibilidade do solo;

$\mathrm{LS}=$ fator topográfico. 
Os limites de perdas toleráveis foram de $6,0 \mathrm{t} \mathrm{ha}^{-1}$ ano $^{-1}$ para Nitossolos e de $12 \mathrm{t} \mathrm{ha}^{-1}$ ano $^{-1}$ para os Latossolos, de acordo com os limites estabelecidos por Bertoni \& Lombardi Neto (1999).

A expectativa de erosão (EE) foi determinada pela equação 7 :

$$
\mathrm{EE}=\mathrm{A}-\mathrm{T} / \mathrm{PNE}
$$

Sendo:

$\mathrm{EE}=$ expectativa de erosão;

$\mathrm{A}=$ perda de solo;

$\mathrm{T}=$ tolerância à perda de solo;

$\mathrm{PNE}=$ potencial natural de erosão.

Os parâmetros considerados foram todos os fatores da equação, e os resultados obtidos pela equação.

Para a análise de agrupamento foi empregada a distância euclidiana simples (DE), utilizando o método de associação de médias (Sneath \& Sokal, 1973) para estruturação dos dendogramas.

Para o cálculo da análise de agrupamento e análise dos componentes principais foi utilizado o programa MINITAB 14 (2002).

\section{RESULTADOS E DISCUSSÃO}

Para o conjunto dos fatores erodibilidade (K); fator topográfico (LS); fator uso e manejo do solo (C) e praticas conservacionistas (P), foram aplicadas as análises de agrupamento e de componentes principais, não tendo sido considerada a erosividade (R) por ela ser constante em toda área. As distâncias euclidianas médias obtidas permitiram elaborar o dendograma, que mostra a formação de grupos e subgrupos heterogêneos, indicando que este conjunto de fatores permite a discriminação de áreas com maiores expectativas de perdas de solo. A análise do dendograma e dos componentes principais confirma os agrupamentos formados, utilizando-se as menores distâncias euclidianas (DE).

Da mesma forma, a aplicação da análise de agrupamento e de componentes principais para os parâmetros resultantes da equação como: perda de solo (A); potencial natural de erosão (PN) e expectativa de erosão (EE) resultou em agrupamentos heterogêneos, permitindo a identificação dos maiores risco de erosão, juntamente com o relevo e manejo do solo.

A uma distancia euclidiana média de 3.52 como perda máxima de similaridade permitiu a formação de 2 grupos (G1 e G2) pela analise dos fatores K, LS, C e P (Figura 3).

O grupo G1 reuniu os fatores representativos para uma classe de solo, Latossolo Vermelho eutrófico, e o grupo G2 os fatores representativos para outra classe de solo, Nitossolo Vermelho eutrófico, com distâncias euclidianas médias de 3,10 e 2,81 respectivamente. 


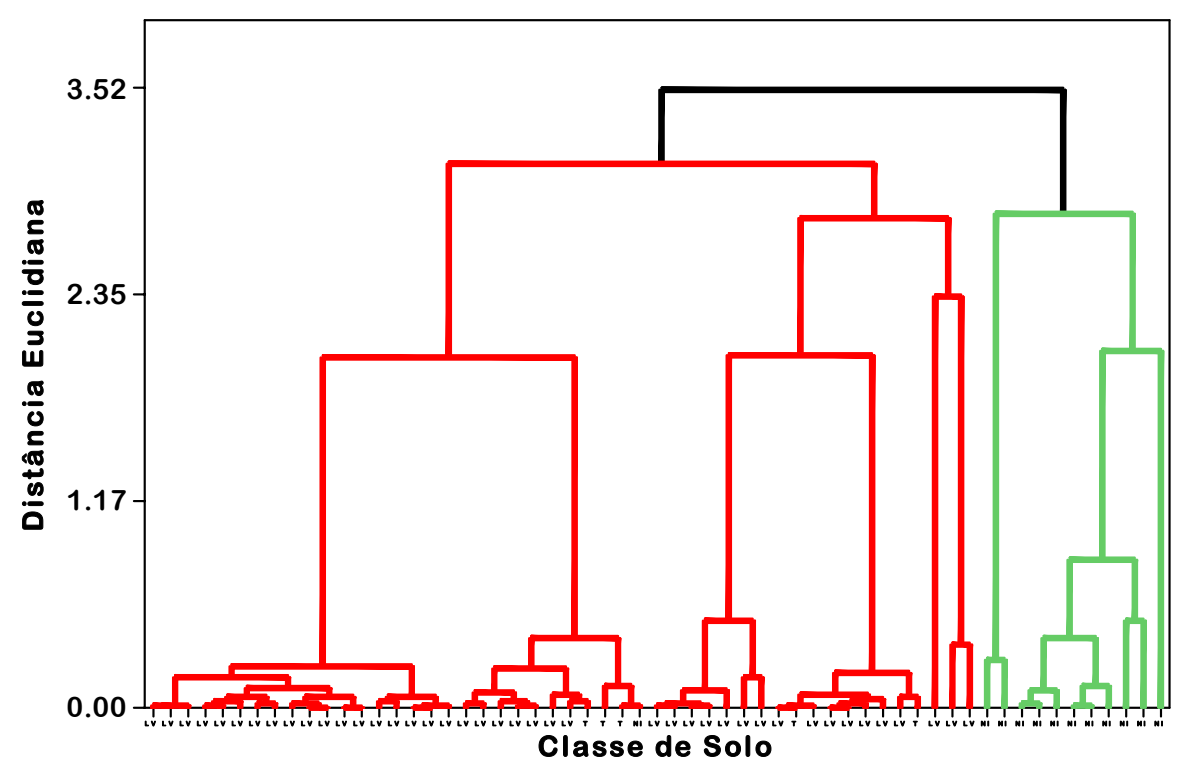

Figura 3. Dendograma para as variáveis $\mathrm{K}, \mathrm{LS}, \mathrm{C}$ e P.

Os valores dos componentes principais ( $\mathrm{Y} 1$ e Y2) obtidos para os fatores $\mathrm{K}, \mathrm{LS}, \mathrm{C}$ e $\mathrm{P}$ possibilitaram a representação destas em um gráfico bidimensional e a definição de seus agrupamentos de acordo com as proximidades de suas localizações. A analise da Figura 4, possibilita verificar que o fator erodibilidade $(\mathrm{K})$ e uso e manejo $(\mathrm{C})$ são influenciados pela classe de solo Nitossolo Vermelho e para os fatores práticas conservacionistas (P) e fator topográfico (LS) são influenciados pela classe de solo Latossolo Vermelho.

Os Nitossolos, geralmente, tem um fator erodibilidade $(\mathrm{K})$ maior, pois apresentam um horizonte $\mathrm{B}$ textural, mais impeditivo à infiltração da água no solo e um perfil menos profundo do que o B latossólico dos Latossolos. Tomazoni et al. (2005) observaram que a erodibilidade varia para cada tipo de solo, pois mesmo que os fatores declividade, precipitação, cobertura vegetal e práticas conservacionistas fossem iguais em solos argilosos e arenosos, os últimos, devido às suas características físicas e químicas, são mais susceptíveis à erosão.

A análise de componentes principais confirma a de agrupamento e os fatores LS, C, K e $\mathrm{P}$ da equação foram sensíveis para discriminar as perdas de solo na área estudada, conforme ilustrado na Figura 4.

No quadro 1 pode-se notar que o primeiro componente principal (Y1) representa 73,8 \% da variância total e o segundo (Y2) 26,1 \% , perfazendo ambos uma variância acumulada de $99,9 \%$. Isso significa que Y1 carrega $73,8 \%$ da informação total fornecida pelos quatro fatores da perda de solo e Y2, 26,1\%, e ambos, 99,9\%.

Pode-se notar, ainda, que os coeficientes de correlação entre os fatores originais e os componentes principais permitem estabelecer para os fatores de perda de solo, a seguinte ordem de importância na formação dos agrupamentos: P, K, LS e C.

A prática conservacionista $(\mathrm{P})$ foi o parâmetro mais importante para refletir a perda de solo, enquanto que o uso atual foi o menos importante. 

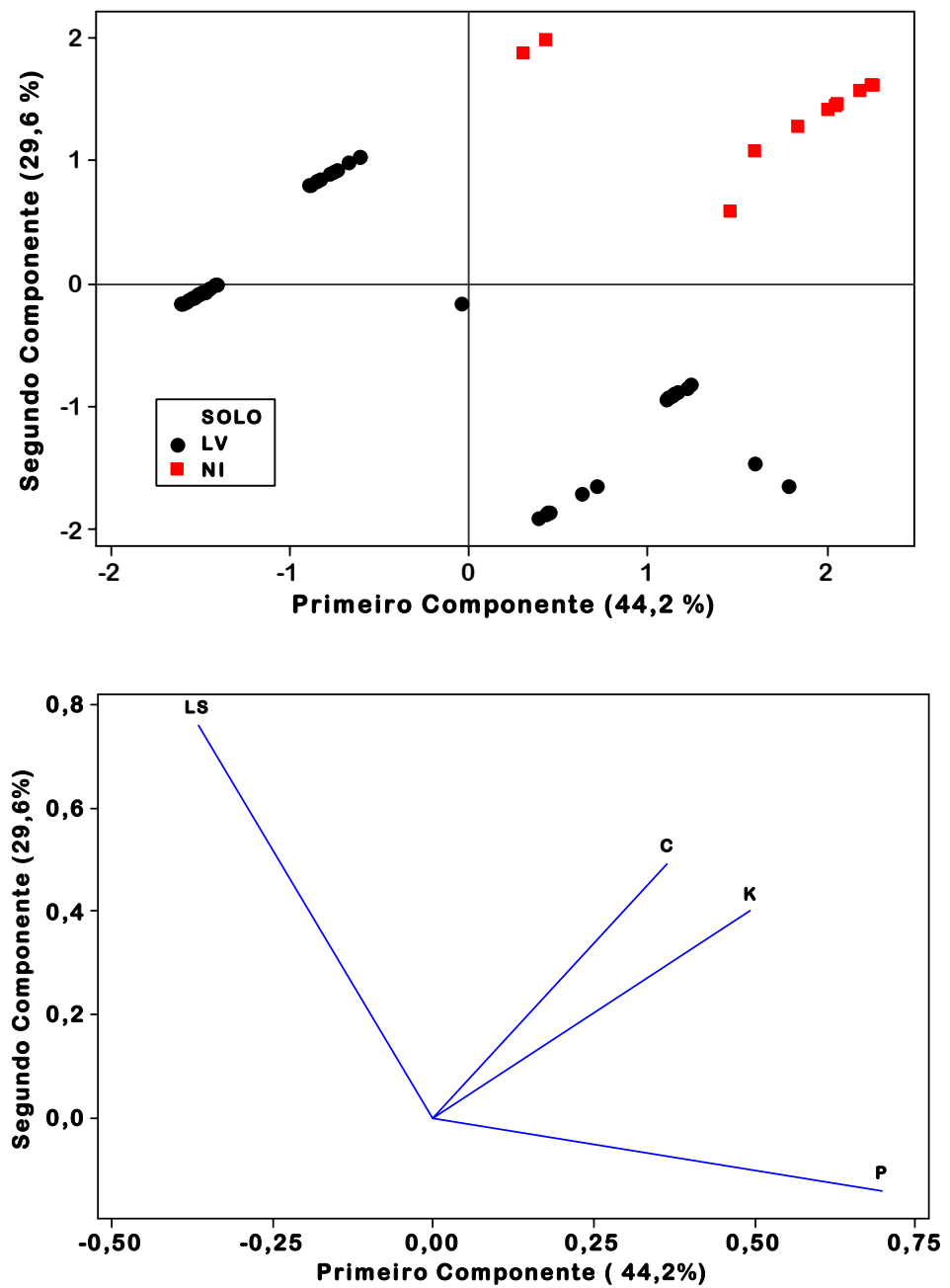

Figura 4. Análise dos componentes principais para os fatores LS, C, K e P.

Tabela 1. Análise de componentes principais, utilizando os quatro fatores da perda de solo para a área de estudo, coeficientes de correlação entre os fatores originais e os dois componentes principais, e ordem de importância dos fatores originais na discriminação e agrupamento das perdas de solo.

\begin{tabular}{|c|c|c|c|}
\hline \multirow[t]{2}{*}{ Fatores } & \multicolumn{2}{|c|}{$\begin{array}{l}\text { Coeficiente de correlação dos } \\
\text { componentes principais }\end{array}$} & \multirow[t]{2}{*}{$\begin{array}{l}\text { Ordem de } \\
\text { importância }\end{array}$} \\
\hline & Y1 & Y2 & \\
\hline $\mathrm{P}$ & 0,700 & 0,142 & $1^{\mathrm{o}}$ \\
\hline K & 0,494 & 0,400 & $2^{o}$ \\
\hline LS & 0,366 & 0,760 & $3^{\circ}$ \\
\hline $\mathrm{C}$ & 0,365 & 0,492 & $4^{o}$ \\
\hline Var. Total (\%) & 73,8 & 26,1 & \\
\hline Var. acumulada (\%) & 73,8 & 99,9 & \\
\hline
\end{tabular}

Para verificar a ação conjunta dos parâmetros, considerando o potencial natural de erosão (PN), perda de solo (A) e a expectativa de erosão (EE), foram aplicadas, novamente, as análises de agrupamento e componentes principais. O dendograma correspondente (Figura 5) 
permite verificar a formação de dois grupos (G1 e G2) constituídos pela similaridade dos parâmetros em relação a $\mathrm{A}, \mathrm{PN}$ e EE , considerando como perda máxima de similaridade a distância euclidiana média de 3,50.

O grupo G1 reuniu os parâmetros do Latossolo Vermelho eutrófico com distância euclidiana média de 2,3 e o G2, os parâmetros do Nitossolo Vermelho eutrófico com distancia euclidiana média de 1,91 .

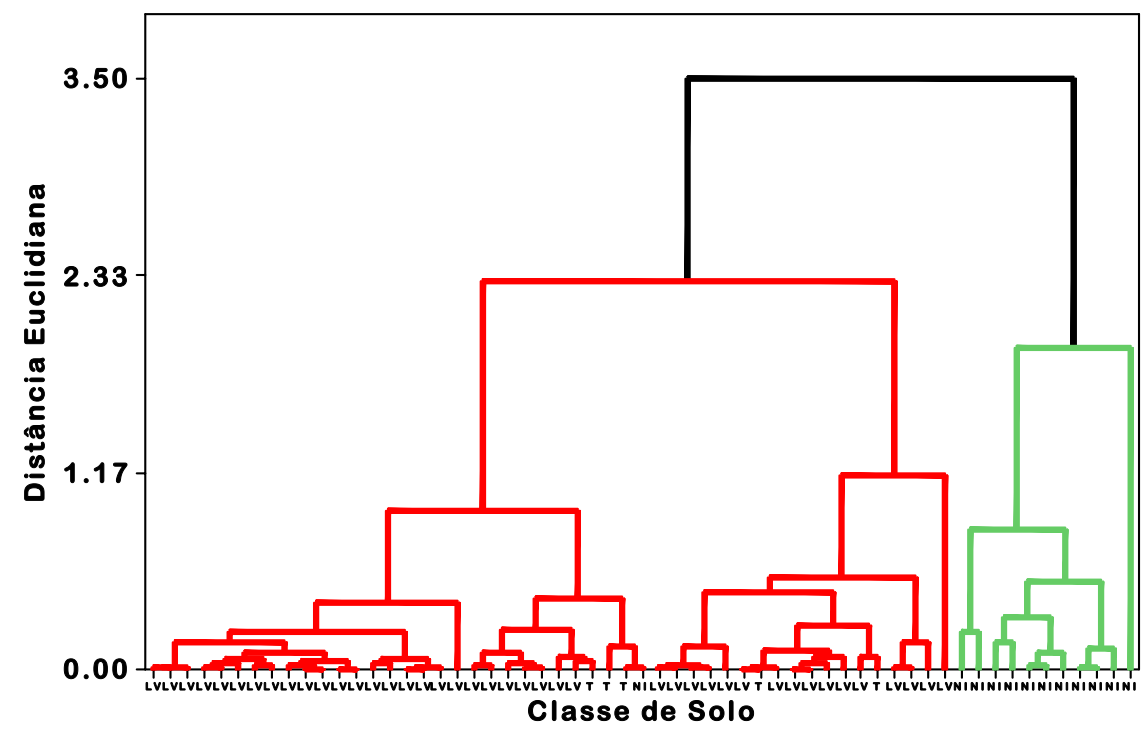

Figura 5. Dendograma para as variáveis PN, A e EE.

A análise de componentes principais possibilitou reunir informações dos três parâmetros através de dois componentes principais Y1 e Y2 e verificar que esta análise confirma a de agrupamento (Figura 6).

A analise da Figura 6, possibilita verificar que o fator EUPS é influenciado pela classe de solo Nitossolo Vermelho (NI) e os fatores PN e EE são influenciados pela classe de solo Latossolo Vermelho (LV).

Na Tabela 2 encontram-se os valores dos coeficientes de correlação entre parâmetros originais e os componentes principais, que mostram que o primeiro componente principal (Y1) representa 98,4 \% da variância total, e o segundo (Y2) 1,6 \%, perfazendo ambos uma variância acumulada de $100 \%$, ou seja, Y1 e Y2 são responsáveis por $100 \%$ das informações fornecidas pelos parâmetros estudados. Os coeficientes de correlação entre os parâmetros originais e os componentes principais permitiram estabelecer a seguinte ordem de importância dos parâmetros na formação dos agrupamentos do risco de erosão: PN, EE e A.

A análise dos resultados destaca a perfeita interação existente entre as características do solo e o relevo no condicionamento do potencial natural de erosão (PN) e perda de solo (A).

Como esses fatores são conseqüências de combinações dos atributos do solo, cultura, manejo e fatores climáticos, estes valores estão associados, primeiramente, a variações intrínsecas das características do solo, principalmente as características ligadas a suscetibilidade a erosão, tais como, textura, permeabilidade e estrutura, e em segundo lugar pelas propriedades extrínsecas, tais como cultivo e manejo do solo. 

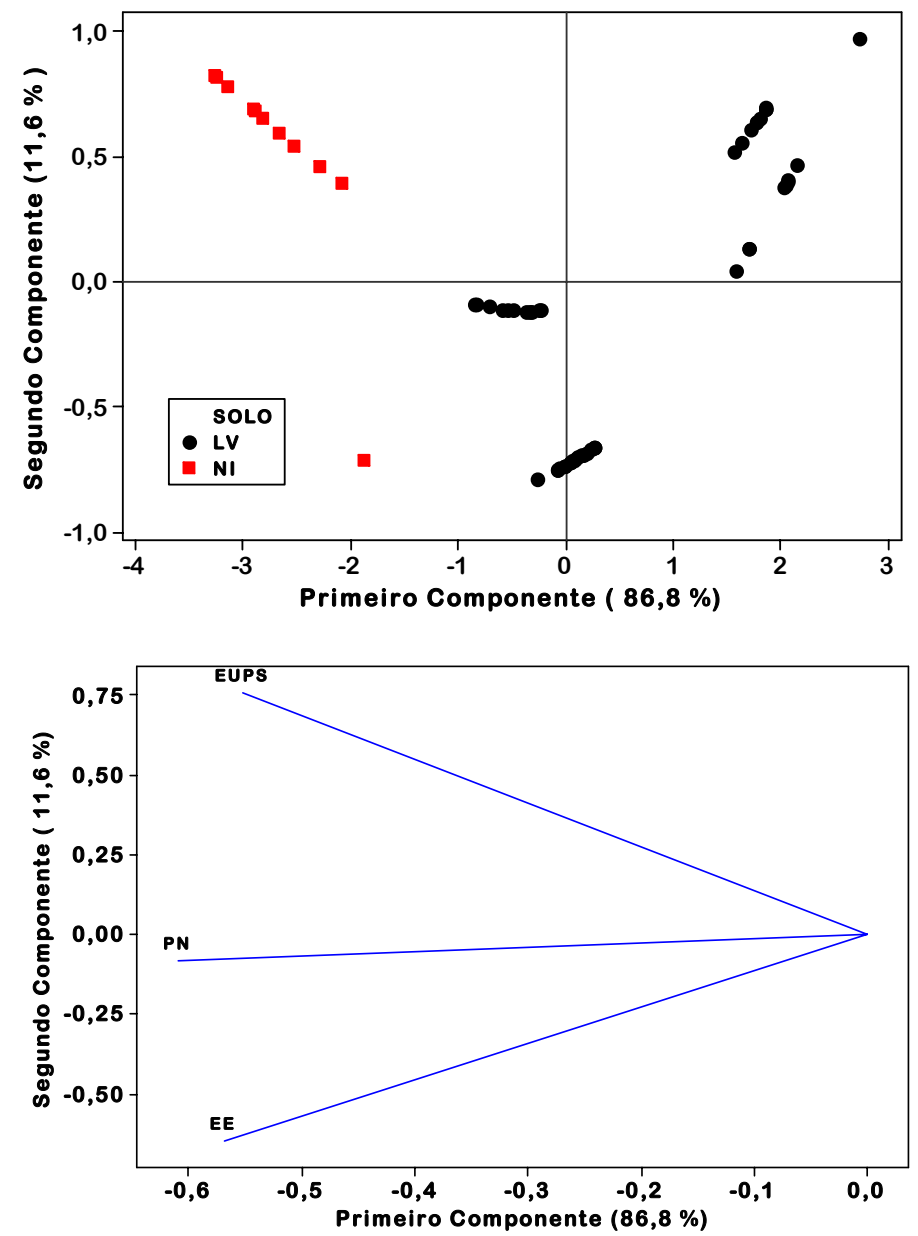

Figura 6. Análise dos componentes principais para os fatores EUPS, PN e EE.

Tabela 2. Análise de componentes principais, utilizando os três parâmetros, coeficientes de correlação entre os parâmetros originais e os dois componentes principais (Y1 e Y2) e ordem de importância dos parâmetros originais na discriminação e agrupamento do risco de erosão.

\begin{tabular}{lccc}
\hline \multirow{2}{*}{ Parâmetros } & \multicolumn{2}{c}{$\begin{array}{c}\text { Coeficiente de correlação dos } \\
\text { componentes principais }\end{array}$} & \multirow{2}{*}{$\begin{array}{c}\text { Ordem de } \\
\text { importância }\end{array}$} \\
\cline { 2 - 3 } & Y1 & Y2 & \\
\hline PN & 0,609 & 0,081 & $1^{\text {o }}$ \\
EE & 0,568 & 0,649 & $2^{\text {o }}$ \\
A & 0,553 & 0,757 & $3^{\text {o }}$ \\
\hline Var. Total (\%) & 98,4 & 1,60 & \\
Var. acumulada (\%) & 98,4 & 100 & \\
\hline
\end{tabular}

\section{Similaridade erosiva}

Áreas onde há ocorrências erosivas totalmente similares entre si tiveram $\mathrm{DE}=2,33 \mathrm{e}$ áreas com inexistência de processos erosivos tiveram $\mathrm{DE}=0,0$. Foram consideradas similares áreas com $\mathrm{DE} \geq 3,50$. 
A aplicação da análise de agrupamento nos pontos da transeção, tendo como parâmetros A, PN e EE, apresentam uma boa associação entre os agrupamentos formados e as diferentes classes de solos, confirmando o potencial destes para delimitar diferentes áreas de risco de erosão.

Dentre os parâmetros A, PN e EE o primeiro grupo (G1) é formado de vários subgrupos, porém, com distância euclidiana menor, destacando maior similaridade entre os subgrupos. Este grupo se destaca pela maior porcentagem dos pontos $(87 \%)$ na transeção, relacionando-se com a ocorrência de Latossolo Vermelho eutrófico, e reflete maior grau de similaridade entre os parâmetros analisados, apesar dos diferentes tipos de manejo, indicando um ambiente mais estável.

O segundo grupo (G2) é formado por um conjunto de amostras (17\%) de maiores valores dos parâmetros avaliados, correlacionando-se a ocorrência de Nitossolo Vermelho eutrófico, localizado na parte de maior altitude do terreno (800 a $820 \mathrm{~m}$ ), com declive mais acentuado, mostrando a influência de fatores relacionados à classe de solo como: textura, estrutura, permeabilidade e fatores conservacionistas e ao relevo, estando de acordo com Novaes Filho et al. (2007), que em estudos em microbacias, concluiram que as alterações de classe de solo em espaços tão pequenos parecem estar mais relacionadas ao relevo e material de origem do que aos outros fatores de formação do solo.

Assim, há indícios que os atuais usos dos solos, ou seja, as culturas e/ou as práticas de manejo, estão contribuindo para o incremente das perdas de solo, principalmente por erosão laminar, nestas áreas de ocorrência do Nitossolo. Pode-se destacar, ainda, que os fatores, cobertura e o manejo do solo se constituem nos mais importantes, já que sua eficiência na redução das perdas do solo por erosão, pode ser quase completa (Schick et al, 2000).

A análise estatística aplicada permitiu a discriminação de dois grupos aliados a duas classes de solos. Dentre os parâmetros estudados, o potencial natural da erosão do solo (PN) mostrou ser um fator importante para determinar os diferentes grupos. Com base na análise discriminante observou-se que $98,4 \%$ dos parâmetros foram corretamente classificados dentro dos grupos e estes possuem potencial erosivo suficiente para uso em programas de manejo e conservação do solo.

\section{CONCLUSÕES}

1. Os indicadores da equação USLE, erodibilidade do solo (K), erosividade da chuva (R), e o Fator topogáfico (LS), e seus resultados possíveis PN, A e EE foram sensíveis para diferenciar as áreas sob Nitossolos daqueles sob Latossolos.

2. A aplicação da técnica de análise multivariada de componentes principais permitiu a distinção das áreas sob Nitossolos e Latossolos.

3. Noventa e oito porcento dos parâmetros analisados foram classificados dentro dos grupos formados.

\section{AGRADECIMENTOS}

Á FUNDUNESP, pelo apoio financeiro (Processo Nº 00218/2003 DFP). 


\section{REFÊRENCIAS}

BERTONI, J.; LOMBARDI NETO, F. Conservação do solo. $3^{\text {a }}$ ed. São Paulo: Ícone, 1999. $355 \mathrm{p}$.

BUENO, C. R. P.; STEIN, D. P. Potencial natural e antrópico de erosão na região de Brotas, Estado de São Paulo. Acta Scientiarum Agronomy, Maringá, v. 26, n. 1, p. 1-5, 2004.

DEMATTÊ, J. A. M.; GARCIA, G. J. Alteration of soil properties through a weathering sequence as evaluated by spectral reflectance. Soil Science Society of America Journal, Madison, v. 63, p. 327-342, 1999.

DEMATTÊ, J. A. M.; NANNI, M. R. Weathering sequence of soils developed from basalt as evaluated by laboratory (IRIS), airborne (AVIRIS) and orbital (TM) sensors. International Journal of Remote Sensing, London, v. 24, p. 4715-4738, 2003.

DENARDIN, J. E. Erodibilidade do solo estimada por meio de parâmetros físicos e químicos. 1990. 81 f. Tese (Doutorado em Agronomia Solos e Nutrição de Plantas) Escola Superior de Agricultura Luiz de Queiroz, Universidade de São Paulo, Piracicaba, 1990.

EMPRESA BRASILEIRA DE PESQUISA AGROPECUÁRIA. Serviço Nacional de Levantamento e Conservação de Solo. Manual de Métodos de análise de solo. Rio de Janeiro, 1997. $112 \mathrm{p}$.

FIDALSKI, J.; TORMENA, C. A.; SCAPIM, C. A. Espacialização vertical e horizontal dos indicadores de qualidade para um Latossolo Vermelho. Revista Brasileira de Ciência do Solo, Viçosa, v. 31, n. 1, p. 9-19, 2007.

GOLDEN SOFTWARE. Surfer for windows:. surface mapping system realese 6.01. Golden, CO, 1995.

INSTITUTO DE PESQUISAS TECNOLÓGICAS DO ESTADO DE SÃO PAULO.

Orientações para o combate à erosão no Estado de São Paulo, bacia do baixo Tietê; áreas de risco à erosão por ravinas e boçorocas e estudo atual da criticidade dos municípios. São Paulo, 1988. v. 1, 66 p. (Relatório 26989).

KRAVCHENKO, A. N. et al. Quantitative mapping of soil drainage classes using topographical data and soil eletrical conductivity. Soil Science Society of America Journal, Madison, v. 66, p. 235-243, 2002.

LAGROTTI, C. A. A. Planejamento agroambiental do município de Santo Antônio do Jardim-SP estudo de caso na microbacia hidrográfica do córrego do Jardim. 2000. $115 \mathrm{f}$. Tese (Doutorado em Engenharia Agrícola) Faculdade de Engenharia Agrícola, Universidade de Campinas, Campinas, 2000. 
LIMA, H.V. et al. Indicadores de qualidade do solo em sistemas de cultivo orgânico e convencional no semi-árido cearense. Revista Brasileira de Ciência do Solo, Viçosa, v. 31 , n. 5, p. 1085-1098, 2007.

MINITAB INCORPORATION. Minitab user's guide. release 14 for Windows. State College,., 2002. Disponível em http://www.minitab.com/en-BR/default.aspx

NOVAES FILHO, J. P. et al. Variabilidade espacial de atributos físicos de solo usada na identificação de classes pedológicas de microbacias na Amazônia Meridional. Revista Brasileira de Ciência do Solo, Viçosa, v. 31, n. 1, p. 91-100, 2007.

RAIJ, B. van et al. Análise química do solo para fins de fertilidade. Campinas: Fundação Cargill, 1987. $170 \mathrm{p}$.

SNEATH, D. H.; SOKAL, R. R. Numerical taxonomy: the principles and practice of numerical classification. San Francisco, W.H. Freeman, 1973. 573 p.

STEIN, D. P. et al.. Potencial de erosão laminar, natural e antrópico, na bacia do PeixeParanapanema. In: SIMPÓSIO NACIONAL DE CONTROLE DE EROSÃO, 4.,1987, Marília. Anais... Marília: ABGE, 1987. p. 105-135.

TOMAZONI, J. C. et al. A sistematização dos fatores da EUPS em SIG para quantificação da erosão laminar na Bacia do Rio Anta gorda (PR). Estudos Geográficos, Rio Claro, v. 3, n. 1, p 1-21, 2005.

SALES, J. S. Análise Comparativa de metodologia para espaçamento entre terraços. 1998. 85 f. Dissertação (Mestrado em Engenharia Agrícola) Faculdade de Engenharia Agrícola, Universidade de Campinas, Campinas, 1998.

SCHICK, J. et al. Erosão hídrica em Cambissolos Húmico alumínico submetido a diferentes sistemas de preparo e cultivo do solo: I. perdas de solo e água. Revista Brasileira de Ciência do Solo, Viçosa, v. 24, n. 2, p. 427-436, 2000.

SPAROVEK, G.; SCHNUG, E. Temporal erosion-induced soil degradation and yield loss. Soil Science Society American Journal, Madison, v. 65, p. 1479-1486, 2001.

VALLADARES, G.S. et al. Análise dos componentes principais e médotos multicritério ordinais no estudo de organossolos e solos afins. Revista Brasileira de Ciência do Solo, Viçosa, v. 32, n. 1, p. 285-296, 2008.

WEBSTER, R.; OLIVER, M. A. Statistical methods in soil and land resource survey. Oxford: Oxford University Press, 1990. chap. 9, p. 147-167.

WISHMEIER, W. H.; SMITH, D. D. Predicting rainfall erosion losses: a guide to conservation planning. Washington DC: Department of Agriculture, 1978. 58 p. (Agriculture handbook, 537). 